\title{
A patient with MEN1 and end-stage chronic kidney disease due to Alport syndrome: Decision making on the eligibility of transplantation
}

\author{
ANTONIO MATRONE ${ }^{1}$, ALESSANDRO BRANCATELLA ${ }^{1}$, PIERO MARCHETTI ${ }^{2}$, ENRICO VASILE ${ }^{3}$, UGO BOGGI ${ }^{4}$, \\ ROSSELLA ELISEI ${ }^{1}$, FILOMENA CETANI ${ }^{5}$, CLAUDIO MARCOCCI ${ }^{5}$, PAOLO VITTI $^{1}$ and FRANCESCO LATROFA ${ }^{1}$ \\ ${ }^{1}$ Department of Clinical and Experimental Medicine, Unit of Endocrinology I, University of Pisa; ${ }^{2}$ Department of Clinical and \\ Experimental Medicine, Division of Metabolism and Cell Transplantation, University of Pisa; ${ }^{3}$ Department of Translational \\ Research and of New Surgical and Medical Technologies, Unit of Oncology, University of Pisa; ${ }^{4}$ Department of Translational \\ Research and of New Surgical and Medical Technologies, Division of General and Transplant Surgery, University of Pisa; \\ ${ }^{5}$ Department of Clinical and Experimental Medicine, Unit of Endocrinology II, University of Pisa, I-56124 Pisa, Italy
}

Received August 20, 2017; Accepted November 28, 2017

DOI: $10.3892 /$ mco.2017.1542

\begin{abstract}
Absence of neoplastic disease in the organ-recipient is required in order to allow organ transplantation. Due to its rarity, no data regarding management of patients with Multiple endocrine neoplasia type 1 (MEN1) and end-stage renal failure candidates for kidney transplantation are available. A 36 year-old man was referred to the present hospital with MEN1, with a neuroendocrine pancreatic tumor and primary hyperparathyroidism and associated Alport syndrome with end stage renal failure. The present study aimed to establish the eligibility of the patient for a kidney transplantation. The neuroendocrine tumor had been treated with duodenopancreatectomy two years earlier and hyperparathyroidism by parathyroidectomy. The review of the literature did not provide data regarding the eligibility for kidney transplantation of patients harboring a neuroendocrine pancreatic tumor in the context of MEN1. Due to the end-stage renal failure, neuroendocrine markers were unreliable and the investigation therefore relied on imaging studies, which were unremarkable. Young age, low-grade tumor, low expression of Ki67, absence of metastatic lymph nodes, onset in the setting of a MEN1 were all positive prognostic factors of the neuroendocrine tumor. Normal serum calcium ruled out persistent primary hyperparathyroidism. Overall, hemodyalisis is known to significantly reduce life expectancy. Benefits of kidney transplantation overcome the risk of neuroendocrine tumor recurrence in a young patient bearing MEN1.
\end{abstract}

Correspondence to: Dr Antonio Matrone, Department of Clinical and Experimental Medicine, Unit of Endocrinology I, University of Pisa, Via Paradisa 2, I-56124 Pisa, Italy

E-mail: anto.matrone@yahoo.com

Key words: MEN1, alport syndrome, transplantation, neuroendocrine tumor, glucagon, hyperparathyroidism, cancer recurrence

\section{Introduction}

Multiple endocrine neoplasia type 1 (MEN1), is a rare autosomal dominant disease, linked to a mutation of a tumor suppressor gene encoding for the protein menin. Tumors occur in parathyroids, pancreas and pituitary, with a variable phenotype. Alport syndrome, a recessive X-linked disease is due to mutations of the genes, encoding for the procollagen IV, usually leading to end-stage chronic kidney disease. Neurosensory deafness, abnormally colored retina and misshapen lenses are the other most relevant features of the syndrome. The patient we describe herein was carrying both these rare diseases. The clinical challenge we had to face was to establish the eligibility of the patient to kidney and possibly pancreas transplantation.

\section{Case report}

We report on a 36 year-old man affected by MEN1, namely with primary hyperparathyroidism (PHPT) and non-secreting neuroendocrine pancreatic tumor and concomitant Alport syndrome with end-stage chronic kidney disease. At the age of 10, following the diagnosis of Alport syndrome in the mother's lineage, the patient underwent a kidney biopsy which showed a histologic picture of Alport syndrome. Five years later, because of increasing proteinuria, angiotensin converting enzyme inhibitor treatment was started. The renal function progressively deteriorated up to end-stage renal failure and, at the age of 32 , hemodialysis was started. The diagnosis of Alport syndrome was confirmed by genetic analysis. Familial PHPT was diagnosed in his father's kindred when the patient was 30 year-old, and MEN1 genetic analysis revealed an uncommon complete deletion of the MEN gene in both the patient and his father. One year later, PHPT was also diagnosed in the patient who underwent surgical removal of the right superior, left superior and left inferior parathyroid glands. After surgery, cinacalcet therapy (30 mg/daily) was started. The patient was referred to our hospital for further evaluation. A whole-body CT scan, performed as part of the work out of 
Table I. Laboratory findings before and after duodenopancreatectomy.

\begin{tabular}{|c|c|c|c|}
\hline & Column 1 (before surgery) & Column 2 (after surgery) & Normal range \\
\hline Glucose (mg/dl) & 73 & $121^{\mathrm{a}}$ & 74-109 \\
\hline Hemoglobin A1c (mmol/mol) & NA & $38^{\mathrm{a}}$ & $20-38$ \\
\hline Chromogranin A (ng/ml) & 1680 & NA & $<100$ \\
\hline Neuron Specific Enolase (ng/ml) & 17.6 & 12.3 & $<12.5$ \\
\hline Glucagon (pg/ml) & 1799 & 298 & $25-250$ \\
\hline Gastrin (pg/ml) & 255 & NA & 25-111 \\
\hline Histamine $(\mathrm{mcmol} / \mathrm{l})$ & 10.6 & NA & $<10$ \\
\hline Calcium (mg/dl) & $10.1^{\mathrm{b}}$ & $8.6-9.7^{\mathrm{c}}$ & $8.6-10.2$ \\
\hline Phosphorus (mg/dl) & 5.8 & 7.3 & $2.7-4.5$ \\
\hline Albumin (g/dl) & 4.58 & 4 & $3,5-5,5$ \\
\hline PTH (pg/ml) & $311^{\mathrm{b}}$ & $248-283^{c}$ & $8-40$ \\
\hline 25 OH Vitamin D (ng/ml) & 16.5 & 8.8 & $30-150$ \\
\hline 1-25 $\mathrm{OH}_{2}$ Vitamin D (pg/ml) & NA & 17 & $20-67$ \\
\hline Bone Alkaline Phosphatase (mcg/l) & 13 & 43 & $2-15$ \\
\hline C-Terminal Telopeptide (ng/ml) & $>6$ & $>6$ & $0.115-0.748$ \\
\hline Osteocalcin (ng/ml) & $>200$ & $>200$ & $6.8-34$ \\
\hline ACTH $(\mathrm{pg} / \mathrm{ml})$ & 31 & NA & $10-50$ \\
\hline Cortisol (mcg/dl) & 13.2 & NA & $6.7-22.6$ \\
\hline IGF-1 (ng/ml) & 166 & NA & $77-250$ \\
\hline GH (ng/ml) & 0.08 & NA & $0.01-3.6$ \\
\hline FSH (mUI/ml) & 5.7 & NA & $1.3-19.5$ \\
\hline $\mathrm{LH}(\mathrm{mUI} / \mathrm{ml})$ & 4.9 & NA & $1.4-12.7$ \\
\hline Testosteron (ng/ml) & 2.7 & NA & $1.75-7.8$ \\
\hline
\end{tabular}

${ }^{\mathrm{a}} \mathrm{After}$ insulin treatment. ${ }^{\mathrm{b}} \mathrm{On}$ cinacalcet and ${ }^{\mathrm{c}} 15$ days after cinacalcet withdrawal. NA, not available.

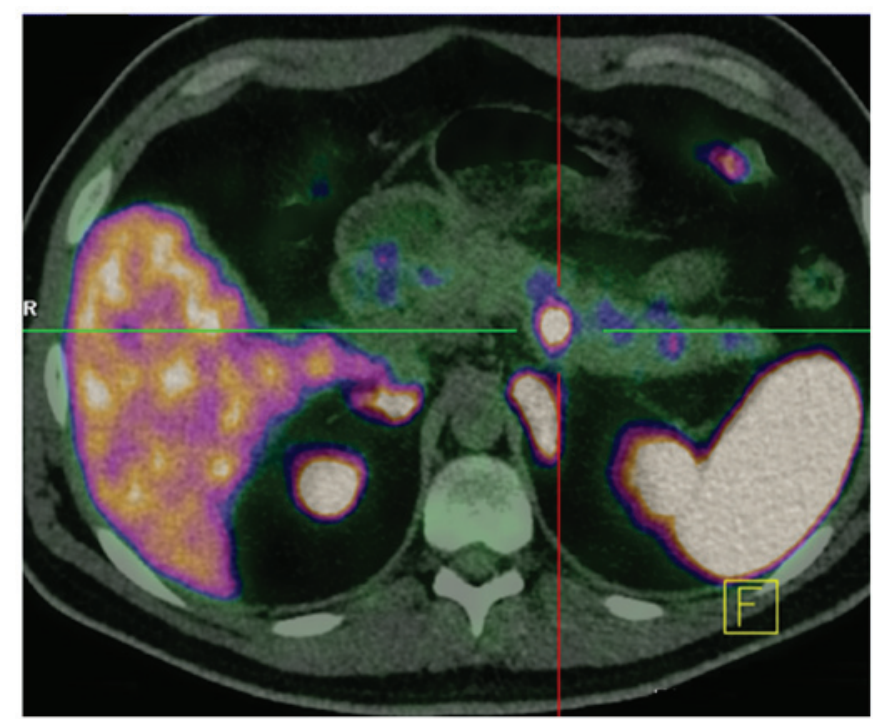

Figure 1.68-Ga-Dotatoc PET scan uptake of pancreatic neuroendocrine lesion.

the MEN1 syndrome, showed multiple pancreatic lesions that were positive at the Gallium-68 Dotatoc PET scan (Fig. 1). Neuroendocrine markers were increased (Table I, column 1). However, treatment with proton pump inhibitor and end-stage renal failure lessened reliability of their measurement. In 2014, at the age of 32 , the patient underwent duodenopancreatectomy plus lymphadenectomy. Histology showed multiple foci of neuroendocrine tumors of intermediate grade $(\mathrm{G} 2)$ widespread in the pancreas, with vascular invasion, 2 mitosis/10 high-power fields, immunostaining positive for $\mathrm{Ki} 67$ (5\%), chromogranin, CD56, insulin and negative for glucagon; no lymph nodes metastases were present (ENETS: pT2N0G2; AJCC: cT1N0M0). After duodenopancreatectomy, treatment with insulin for secondary diabetes was started. After recovering from pancreatic surgery, in 2016, at the age of 36 years, the patient was evaluated for eligibility to kidney and possibly pancreas transplantation. At admission he was in good conditions. Daily treatment consisted of cinacalcet $30 \mathrm{mg}$, sevelamer 4,800 mg, lispro $30 \mathrm{IU}$, degludec $6 \mathrm{IU}$, pancreolipase 60,000 IU, cardioaspirin $100 \mathrm{mg}$, esomeprazol $40 \mathrm{mg}$. Laboratory findings are shown in Table I (column 2). Neck ultrasound and tc-99m sestamibi scan showed a hyperplastic right inferior parathyroid (diameter: $20 \mathrm{~mm}$ ). Abdominal CT scan, Gallium-68 Dotatoc PET scan and pituitary MRI were all unremarkable.

\section{Discussion}

MEN1, an autosomal dominant disease, presents with a low incidence (2-4/1,000,000 cases per year) but a very high penetrance. It is linked to a mutation of a tumor suppressor 
gene on the long arm (q) of chromosome 11 at position 13.1 encoding for the protein menin. Tumors occur in parathyroids, pancreas and pituitary, with a variable phenotype. Alport syndrome, a recessive X-linked disease with an incidence of $1 / 50,000$ per year, is due to mutations of the COL4A3, A4 and $A 5$ genes, encoding for the procollagen IV. Neurosensory deafness, abnormally colored retina and misshapen lenses are the most relevant features of the syndrome. The patient we describe herein was carrying both these rare diseases. The clinical challenge we had to face was to establish the eligibility of the patient to kidney and possibly pancreas transplantation. To address this question we had to take into account several considerations, that will be addressed thereafter. It is generally agreed that only patients with no evidence of tumor are eligible to organ transplantation. We had therefore to establish that the neuroendocrine pancreatic tumor was on remission, on the basis of undetectable serum markers and negative imaging. Unfortunately, we could not rely on measurement of chromogranine because of coexistent modifiable (PPI treatment) and non-modifiable (end-stage chronic kidney disease) interfering factors. Measurement of glucagon, which was very high before duodenopancreatectomy, was not informative for several reasons. Indeed, hyperglucagonemia can be observed in end stage renal failure (1), glucagon might have an extra-pancreatic origins and, in addition, the results of its measurement are often discordant (2). Older age, high-grade tumor, high expression of Ki67, number of metastatic lymph nodes and tumor-related symptoms are all negative prognostic factors for the recurrence of neuroendocrine tumors (3). Our patient was young and presented with a low/intermediate malignancy grade tumor with low grade of Ki67 expression. It has been reported that pancreatic neuroendocrine tumors in patients with MEN1 have a better prognosis compared to sporadic forms, even in case of recurrence (4). Moreover, because imaging studies were negative two years after duodenopancreatectomy, we concluded that the likelihood that the pancreatic tumor was on remission was high. The outcome of cancer in remission in patients undergoing organ transplantation is uncertain. A recent meta-analysis of all-organ transplanted patients has shown a higher mortality rate (HR 3.13 ; 95\% CI, 2.29-4.27) and a higher risk of other neoplasia (HR 1.92; 95\% CI, 1.52-2.42) in those cured from a concomitant cancer than in transplanted, non-neoplastic patients (5). In keeping with their low prevalence, no data on the prevalence of neuroendocrine tumors in all-organ transplanted patients has been reported. Available data indicates that the recurrence rate of cancer in transplanted patients ranges between 5 and $21 \%$, and is strictly related to the lag between cancer remission and kidney transplantation as well as type of tumors, multiple myeloma, breast cancer and kidney cancer showing the higher recurrence rate (6). The suggested lag between cancer remission and eligibility to kidney transplantation varies according to the different guidelines and depends upon to the type of the tumor. No data about patients with neuroendocrine tumors undergoing kidney transplantation are currently available. One should also take in mind that the mortality rate, particularly because of cardiovascular complications, is higher in hemodialyzed patients than in the general population. According to a recent report, the duration of hemodialysis influences the outcome of patients with end-stage kidney disease (7).
Because our patient was on hemodialysis for 3 years, his life expectancy was reduced by $8-20 \%$ (7). In addition, chronic kidney disease and dialysis are independent risk factors for the occurrence of new tumors (8), in particular lung and urinary tract cancer (9). We did not consider pancreatic transplantation as urgent as kidney transplantation because hyperglicemia was well managed by insulin treatment. In addition, compared to kidney transplantation, pancreatic transplantation requires a higher immunosuppressive treatment, which could negatively affect the outcome of the neuroendocrine pancreatic tumor. Immunosuppressive therapy by itself increases tumor incidence and recurrences, the longer the treatment the higher the incidence of tumors (10). Finally, while establishing eligibility to kidney transplantation, we had to take into consideration the concomitant hyperparathyroidism. The finding of normal serum calcium and high PTH fifteen days after the withdrawal of cinacalcet was in keeping with the end-stage renal disease and ruled out persistent primary hyperparathyroidism. Therefore an additional parathyroidectomy was not taken into consideration. In addition, post-surgical hypoparathyroidism usually worsens the adynamic bone state typical of uremic patients (11) and requires a strict adherence to medications to avoid hypocalcemia. We foresaw that a possible further rise in serum calcium would require a medical treatment in order to defer parathyroidectomy as long as possible. We concluded in favor of the eligibility of the patient for kidney transplantation for the following reasons: i) the remission of the pancreatic tumor ant its low risk of recurrence; ii) the higher mortality in end-stage renal failure overcomes the increased risk of recurrence of the pancreatic tumor after kidney transplantation; and iii) no evidence of hypercalcemia.

\section{Acknowledgements}

A.M. contributed to this paper as recipient of the $\mathrm{PhD}$ program in Clinical and Translational Science of the University of Pisa. We would like to thank Ministero dell' Istruzione, dell' Università e della Ricerca (MIUR, investigator grant 2015 project code PRIN 2015HPMLFY) for the continuing research support.

\section{References}

1. Bilbrey GL, Faloona GR, White MG and Knochel JP: Hyperglucagonemia of renal failure. J Clin Invest 53: 841-847, 1974.

2. Wewer Albrechtsen NJ, Hartmann B, Veedfald S, Windeløv JA, Plamboeck A, Bojsen-Møller KN, Idorn T, Feldt-Rasmussen B, Knop FK, Vilsbøll T, et al: Hyperglucagonaemia analysed by glucagon sandwich ELISA: Nonspecific interference or truly elevated levels? Diabetologia 57: 1919-1926, 2014.

3. Falconi M, Eriksson B, Kaltsas G, Bartsch DK, Capdevila J, Caplin M, Kos-Kudla B, Kwekkeboom D, Rindi G, Klöppel G, et al: ENETS consensus guidelines update for the management of patients with functional pancreatic neuroendocrine tumors and non-functional pancreatic neuroendocrine tumors. Neuroendocrinology 103: 153-171, 2016.

4. Rindi G, Falconi M, Klersy C, Albarello L, Boninsegna L, Buchler MW, Capella C, Caplin M, Couvelard A, Doglioni C, et al: TNM staging of neoplasms of the endocrine pancreas: Results from a large international cohort study. J Natl Cancer Inst 104: 764-777, 2012.

5. Acuna SA, Huang JW, Daly C, Shah PS, Kim SJ and Baxter NN: Outcomes of solid organ transplant recipients with preexisting malignancies in remission: A systematic review and meta-analysis. Transplantation 101: 471-481, 2017. 
6. Chapman JR, Sheil AG and Disney AP: Recurrence of cancer after renal transplantation. Transplant Proc 33: 1830-1831, 2001.

7. Orandi BJ, Luo X, Massie AB, Garonzik-Wang JM, Lonze BE, Ahmed R, Van Arendonk KJ, Stegall MD, Jordan SC, Oberholzer J, et al: Survival benefit with kidney transplants from HLA-incompatible live donors. N Engl J Med 374: 940-950, 2016.

8. Vajdic CM, McDonald SP, McCredie MR, van Leeuwen MT, Stewart JH, Law M, Chapman JR, Webster AC, Kaldor JM and Grulich AE: Cancer incidence before and after kidney transplantation. JAMA 296: 2823-2831, 2006.

9. Wong G, Hayen A, Chapman JR, Webster AC, Wang JJ, Mitchell P and Craig JC: Association of CKD and cancer risk in older people. J Am Soc Nephrol 20: 1341-1350, 2009.
10. Mortaz E, Tabarsi P, Mansouri D, Khosravi A, Garssen J Velayati A and Adcock IM: Cancers related to immunodeficiencies: Update and perspectives. Front Immunol 7: 365, 2016.

11. Drüeke TB and MassyZA: Changing bone patterns with progression of chronic kidney disease. Kidney Int 89: 289-302, 2016. 